D) Check for updates

Cite this: Chem. Commun., 2022, 58,795

Received 3rd November 2021 Accepted 8th December 2021

DOI: 10.1039/d1cc06195j

rsc.li/chemcomm

\section{One-step covalent hydrophobic/hydrophilic functionalization of chemically exfoliated molybdenum disulfide nanosheets with RAFT derived polymers $\dagger$}

\author{
Andriana Plantzopoulou, ${ }^{a}$ Anastasios Stergiou, (D) ${ }^{a}$ Martha Kafetzi, ${ }^{a}$ \\ Raul Arenal, (D) *bcd Stergios Pispas (D) *a and Nikos Tagmatarchis (D) *a
}

The covalent functionalization of chemically exfoliated molybdenum disulfide (ce-MoS 2 ) with hydrophobic poly(methyl methacrylate) and hydrophilic poly(acrylic acid) polymers, in a single-step without additives, is presented. The nature of chemical modification and the impact on the structure of ce-MoS 2 were spectroscopically investigated. Complexation of $\mathrm{Eu}^{3+}$ was accomplished on grafted polycarboxylate chains on $\mathrm{MoS}_{2}$.

Molecular functionalization of exfoliated $\mathrm{MoS}_{2}$ nanosheets has gained increased interest. ${ }^{1-6}$ In particular, chemically exfoliated $\mathrm{MoS}_{2}$ nanosheets (ce-MoS ) derived by treating the bulk semiconducting $\mathrm{MoS}_{2}$ powder with organolithium compounds ${ }^{7}$ are a proven candidate towards stable covalently modified nanosheets with small molecules. ${ }^{6,8-10}$ However, covalent functionalization of ce-MoS $\mathrm{M}_{2}$ nanosheets with polymers remains in its infancy. Recently, via a 2-step approach, poly(perfluoralkyl) chains have been covalently grafted by combining diazonium chemistry followed by a free-radical polymerization. ${ }^{11}$ Reversible addition fragmentation transfer (RAFT) polymerization is reportedly utilized in multi-step strategies as a chemical tool towards covalent $\mathrm{MoS}_{2}$-polymer ensembles. ${ }^{12-14}$ Nevertheless, insights into the reaction mechanisms taking place between the ce- $\mathrm{MoS}_{2}$ nanosheets and RAFTderived polymers, as well as the impact on the structure of the nanosheets are missing or insufficiently described.

In this communication, we present the covalent functionalization of ce- $\mathrm{MoS}_{2}$ with hydrophobic poly(methyl methacrylate),

\footnotetext{
${ }^{a}$ Theoretical and Physical Chemistry Institute, National Hellenic Research Foundation, 48 Vassileos Constantinou Avenue, Athens 11635, Greece. E-mail: tagmatar@eie.gr

${ }^{b}$ Laboratorio de Microscopias Avanzadas (LMA), Universidad de Zaragoza, Mariano Esquillor s/n, Zaragoza 50018, Spain

${ }^{c}$ Instituto de Nanociencia y Materiales de Aragon (INMA), CSIC-U. de Zaragoza, Calle Pedro Cerbuna 12, Zaragoza 50009, Spain

${ }^{d}$ ARAID Foundation, Zaragoza 50018, Spain

$\dagger$ Electronic supplementary information (ESI) available: Synthetic procedures, UV-vis spectra, digital photos, spatial Raman spectroscopy mapping and TEM images. See DOI: 10.1039/d1cc06195j
}

PMMA, and hydrophilic poly(acrylic acid), PAA, polymers derived by RAFT polymerization. The reaction proceeds in a single step in aqueous media without additives. The major advantage of the method is the chemical coupling of well-defined and characterized counterparts in a single step, in contrast to multi-step routes and uncontrolled in situ radical polymerization reactions. We investigated the nature of the chemical modification spectroscopically by FT-IR and spatial Raman spectroscopy mapping, shedding light on the impact of functionalization on the structure of ce- $\mathrm{MoS}_{2}$. Moreover, exploiting the polycarboxylate nature of $\mathrm{MoS}_{2}-\mathrm{PAA}$ nanosheets and their superior stability in water, we investigated the complexation dynamics for water-soluble fluorescent $\mathrm{Eu}^{3+}$ with the aid of photoluminescence (PL) spectroscopy.

To start with, ce- $\mathrm{MoS}_{2}$ was prepared via chemical intercalation with $n$-butyllithium, followed by ultrasonic-assisted exfoliation in distilled water. The success of the intercalation/ exfoliation process was initially assessed via UV-vis spectroscopy (Fig. S1, ESI $\dagger$ ), where the characteristic excitonic transitions of the semiconducting $2 \mathrm{H}$ (trigonal prismatic) bulk $\mathrm{MoS}_{2}$ were absent, suggesting symmetry transition to the octahedral $1 \mathrm{~T}-\mathrm{MoS}_{2}$ phase. Specifically, the recorded electronic absorption features located at 250 and $305 \mathrm{~nm}$ are characteristic of the metallic $1 \mathrm{~T}-\mathrm{MoS}_{2}$ polytype and also verify that the prepared ce$\mathrm{MoS}_{2}$ nanosheets have surface sulfur sites with electron surplus stabilized by lithium cations (Fig. 1a). Freshly prepared oxygenprotected ce-MoS $\mathrm{M}_{2}$ suspensions were used for the covalent decoration of the nanosheets with RAFT-derived PMMA and PAA polymer chains to fully exploit these highly reactive electron-rich exfoliated $\mathrm{MoS}_{2}$ nanosheets. Notably, RAFTderived polymers retain the thiocarbonylthio group of the utilized chain transfer agent (CTA) or RAFT agent at their $\omega$-terminus constituting dormant macro-RAFT agents, being suitable for synthesizing block copolymers, and preparing end-functional polymers via re-activation of the thiocarbonylthio groups. ${ }^{15,16}$ In this context, ce- $\mathrm{MoS}_{2}$, possessing electron surplus, was utilized for the $\omega$-terminal functionalization of RAFT-derived PMMA and PAA polymers towards covalently 
a)

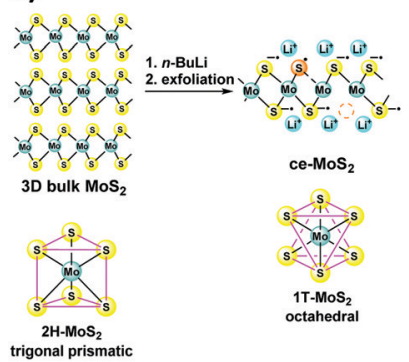

b)

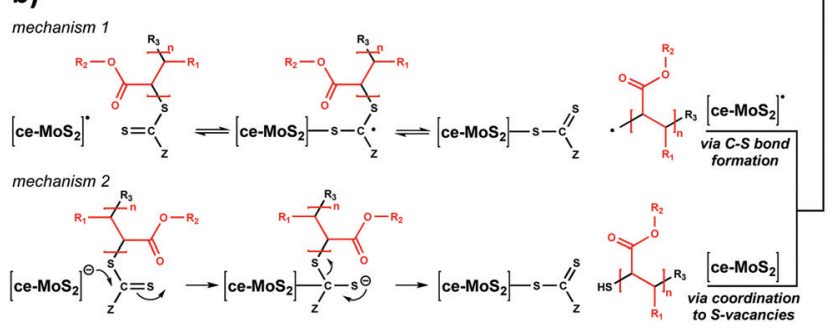

Fig. 1 Schematic illustration of the (a) chemical exfoliation of the bulk $2 \mathrm{H}-\mathrm{MoS}_{2}$ to $1 \mathrm{~T}$ ce- $\mathrm{MoS}_{2}$ and subsequent covalent functionalization with PMMA and PAA polymer chains. (b) Proposed reaction mechanisms furnishing $\mathrm{MoS}_{2}-\mathrm{PMMA}$ and $\mathrm{MOS}_{2}-\mathrm{PAA}$ ensembles.

formed $\mathrm{MoS}_{2}$-PMMA and $\mathrm{MoS}_{2}-\mathrm{PAA}$ ensembles (Fig. 1a). Two main reaction mechanisms are expected to compete: (i) radical and/or (ii) nucleophilic attack to the $\omega$-terminal thiocarbonylthio group of the polymer chain, by reactive radical and/or negatively charged S-sites on the surface of ce- $\mathrm{MoS}_{2}$, respectively. In the case of a radical-mediated reaction, radicals centred around S-defects ${ }^{17}$ at the surface of ce-MoS $\mathrm{M}_{2}$ attack the $\mathrm{S}$-atom of the $\mathrm{C}=\mathrm{S}$ bond of the thiocarbonylthio group releasing polymer bound radicals, which are trapped by neighbouring radicals residing on the surface of ce- $\mathrm{MoS}_{2}$ (mechanism 1, Fig. 1b). According to the second mechanism, reactive nucleophilic anion species centred at S-atoms at the surface of ce- $\mathrm{MoS}_{2}$ attack the C-atom of the $\mathrm{C}=\mathrm{S}$ bond of the thiocarbonylthio group releasing thiol-terminated polymer chains, which are then coordinated to S-vacant sites at the surface of ce- $\mathrm{MoS}_{2}$ (mechanism 2, Fig. 1b). To perform the reaction, a waterisopropanol suspension of the freshly prepared ce- $\mathrm{MoS}_{2}$ was mildly heated at $60{ }^{\circ} \mathrm{C}$ in the presence of PMMA under oxygenfree conditions. PMMA was also selected as a candidate owing to its functional groups, e.g. methyl esters, which are unable to develop electrostatic interactions with the surface of $\mathrm{MoS}_{2}$. The newly prepared $\mathrm{MoS}_{2}$-PMMA was excessively purified by repeating dispersion/isolation cycles to ensure the removal of trapped and physiosorbed polymer chains, within the nanosheets.

The IR spectra of $\mathrm{MoS}_{2}-\mathrm{PMMA}$ revealed the characteristic ester $\mathrm{C}=\mathrm{O}$ stretching mode at $1721 \mathrm{~cm}^{-1}$, appearing as a broad band, as well as the ester $\mathrm{C}-\mathrm{O}$ stretching mode centred at $1140 \mathrm{~cm}^{-1}$, arising from the covalently grafted PMMA chains (Fig. 2a). In previous reports, it was proposed that polymer chains can be grafted onto ce- $\mathrm{MoS}_{2}$ in the form of thiols ${ }^{14}$ and alkylthiol-based linkers ${ }^{12}$ and with ce- $\mathrm{MoS}_{2}$ acting as the Z-functionality of the CTA ( $c f$. Fig. 1). ${ }^{13}$ Inevitably, the presence a)

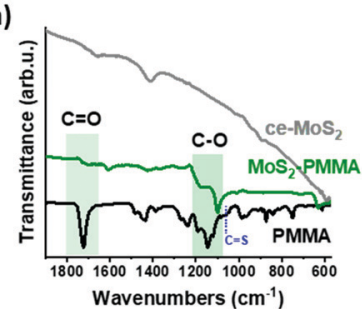

b)

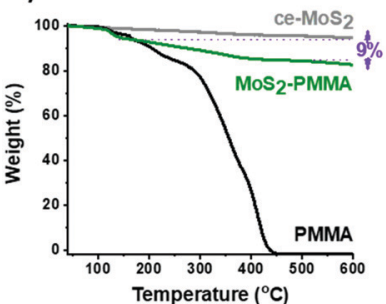

c)

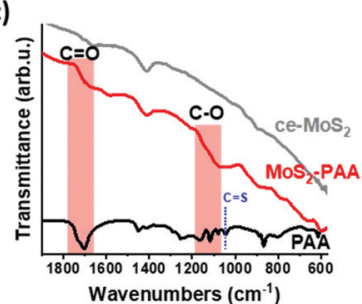

d)

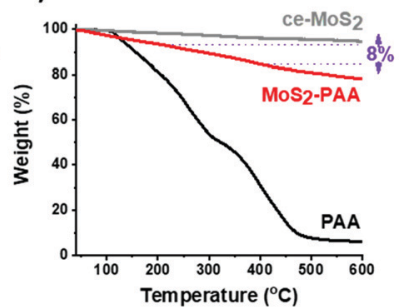

Fig. 2 FT-IR spectra for (a) ce-MoS 2 (grey), PMMA (black) and $\mathrm{MoS}_{2}-$ PMMA (green), and (c) ce-MoS 2 (grey), PAA (black) and $\mathrm{MoS}_{2}-\mathrm{PAA}$ (red). TGA graphs for (b) ce-MoS 2 (grey), PMMA (black) and $\mathrm{MoS}_{2}-\mathrm{PMMA}$ (green), and (d) $c e-\mathrm{MoS}_{2}$ (grey), PAA (black) and $\mathrm{MoS}_{2}-\mathrm{PAA}$ (red).

of the newly formed $\mathrm{C}-\mathrm{S}$ bonds between the nanosheets and polymers was indiscernible in the IR spectra. The same stands true for our study. It is, however, noteworthy that the $\mathrm{C}=\mathrm{S}$ bond, originating from the thiocarbonylthio group, was not present in the IR spectrum of $\mathrm{MoS}_{2}$-PMMA. It is reasonable to consider that, due to the reductive environment, the survival of surface disulfide Mo-S-S- $(\mathrm{C}=\mathrm{S})-\mathrm{Z}$ species produced via both the proposed mechanisms is unlikely.

Thermogravimetric analysis (TGA) under a nitrogen atmosphere for $\mathrm{MoS}_{2}$-PMMA verified a weight loss in the region of 200-400 ${ }^{\circ} \mathrm{C}$ as high as $9 \%$, arising from the grafted polymer chains (Fig. 2b). A weight loss at a low temperature, i.e. $<150{ }^{\circ} \mathrm{C}$, was not observed; thus the presence of Mo-S-S$(\mathrm{C}=\mathrm{S})-\mathrm{Z}$ species in the $\mathrm{MoS}_{2}$-PMMA ensembles is excluded. Therefore, from the FT-IR and TGA studies, we conclude that the surface of the nanosheets is exclusively decorated by the polymeric carbon backbone.

Additional evidence for the covalent insertion of PMMA chains onto $\mathrm{MoS}_{2}$ was provided by investigating the stability of $\mathrm{MoS}_{2}$-PMMA dispersions in water, toluene, methanol and dichloromethane. Interestingly, $\mathrm{MoS}_{2}$-PMMA was found to form the most stable dispersion in toluene, which is an excellent hydrophobic solvent for solubilizing the PMMA polymer, while, in water, which is an excellent solvent to exfoliate and store ce-MoS $\mathrm{Mo}_{2}$, no stable dispersion of $\mathrm{MoS}_{2}$-PMMA was formed (Fig. S2, ESI $\dagger$ ). Then, we employed PAA as a hydrophilic polymer counterpart for the covalent modification of ce- $\mathrm{MoS}_{2}$ for generating aqueous-stable $\mathrm{MoS}_{2}$-PAA ensembles. The preparation and characterization of $\mathrm{MoS}_{2}-\mathrm{PAA}$ were conducted as previously described. Briefly, the FT-IR spectra of the isolated $\mathrm{MoS}_{2}$-PAA displayed the characteristic carboxylic acid $\mathrm{C}=\mathrm{O}$ stretching mode at $1704 \mathrm{~cm}^{-1}$, as well as the carboxylic acid $\mathrm{C}-\mathrm{O}$ stretching mode centred at $1116 \mathrm{~cm}^{-1}$, originating from the grafted PAA polymer chains (Fig. 2c). From the TGA 
thermograms, an $8 \%$ weight loss in the region of $200-400{ }^{\circ} \mathrm{C}$ was observed, indicating the presence of the PAA chains (Fig. 2d), while $\mathrm{MoS}_{2}$-PAA was found to form highly stable dispersions in water, which is an excellent solvent for both ce$\mathrm{MoS}_{2}$ and PAA components (Fig. S3, ESI $\dagger$ ).

In addition, the impact of covalent functionalization on the structure of ce- $\mathrm{MoS}_{2}$ was evaluated with the aid of spatial Raman spectroscopy under 514 and $633 \mathrm{~nm}$ laser excitation. For this, 196 spectra collected from a $20 \times 20 \mu \mathrm{m}$ area were analysed. The octahedral $1 \mathrm{~T}$ symmetry of ce- $\mathrm{MoS}_{2}$ was validated by the presence of the characteristic $\mathrm{J}_{1}, \mathrm{~J}_{2}$, and $\mathrm{J}_{3}$ phonon modes located at 151, 221 and $329 \mathrm{~cm}^{-1}$, in both 514 and $633 \mathrm{~nm}$ laser lines (Fig. 3a and b). Interestingly, the J modes are conserved in the functionalized $\mathrm{MoS}_{2}-\mathrm{PMMA}$ and $\mathrm{MoS}_{2}$-PAA materials manifesting that the particular covalent functionalization has a stabilizing effect on the metastable octahedral symmetry of $\mathrm{MoS}_{2}$. Furthermore, under $514 \mathrm{~nm}$ excitation, an increased fullwidth-half-maximum (FWHM) in the $\mathrm{A}_{1 \mathrm{~g}}$ mode $\left(\sim 404 \mathrm{~cm}^{-1}\right)$ of ce-MoS $\mathrm{MoS}_{2}$-PMMA and $\mathrm{MoS}_{2}$-PAA was evident, as compared to the bulk $\mathrm{MoS}_{2}$, indicating the presence of few layers. This was further elucidated by calculating the frequency difference of $\mathrm{A}_{1 \mathrm{~g}}-\mathrm{E}^{1}{ }_{2 \mathrm{~g}}$ modes under $514 \mathrm{~nm}$ excitation. For the bulk $2 \mathrm{H}$ $\mathrm{MoS}_{2}$, a $26 \mathrm{~cm}^{-1}$ value was registered, while for the ce- $\mathrm{MoS}_{2}$ nanosheets the corresponding value was $23 \mathrm{~cm}^{-1}$, implying the
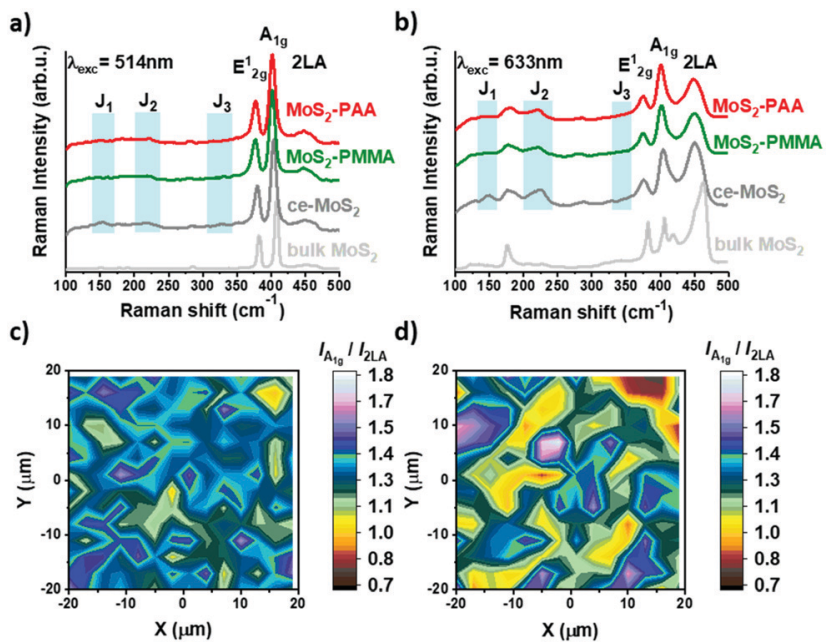

d)

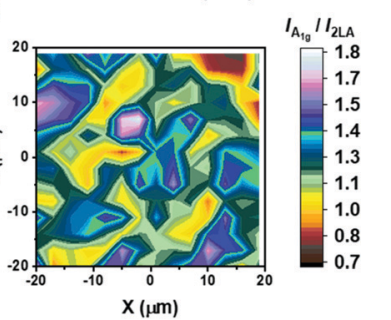

e)
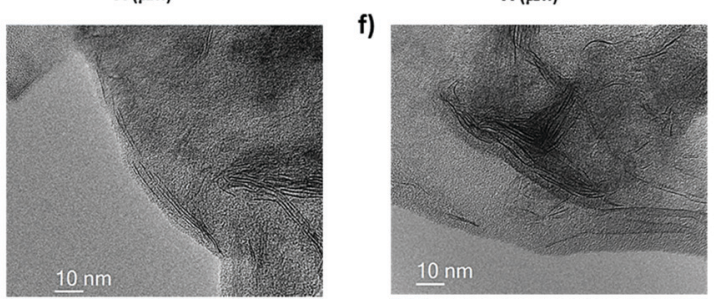

Fig. 3 Representative Raman spectra (an average of 196 spectra from a $20 \times 20 \mu \mathrm{m}$ area) under (a) $514 \mathrm{~nm}$ and (b) $633 \mathrm{~nm}$ laser excitations at a low power $\left(0.3 \mathrm{~mW} \mathrm{~cm}^{-2}\right)$ and under ambient conditions for the bulk $\mathrm{MoS}_{2}$ (light grey), ce-MoS 2 (grey), MoS 2 PMMA (green) and $\mathrm{MoS}_{2}-\mathrm{PAA}$ (red). Spatial Raman maps of $20 \times 20 \mu \mathrm{m}$ under $633 \mathrm{~nm}$ at a low power $\left(0.3 \mathrm{~mW} \mathrm{~cm}^{-2}\right)$ and under ambient conditions for (c) $\mathrm{MoS}_{2}-\mathrm{PMMA}$ and (d) $\mathrm{MOS}_{2}-\mathrm{PAA}$. Scale bar represents the value of the $\mathrm{A}_{1 \mathrm{~g}} / 2 \mathrm{LA}$ intensity ratio. Exemplary HRTEM images of (e) $\mathrm{MoS}_{2}-\mathrm{PMMA}$ and (f) $\mathrm{MoS}_{2}-\mathrm{PAA}$. delamination of the three-dimensional superstructure towards few-layered nanosheets ( $<8$ layers). Notably, the $\mathrm{MoS}_{2}-\mathrm{PMMA}$ and $\mathrm{MoS}_{2}-\mathrm{PAA}$ ensembles displayed $\mathrm{A}_{1 \mathrm{~g}}-\mathrm{E}^{1}{ }_{2 \mathrm{~g}}$ values reaching a maximum value of $24 \mathrm{~cm}^{-1}$; thus, the covalent functionalization proved to shield the nanosheets against restacking to multi-layered non-functionalized structures. The proof of the chemical incorporation of the polymer chains on the surface of the ce- $\mathrm{MoS}_{2}$ nanosheets was given by spatial Raman spectroscopy mapping of the $\mathrm{MoS}_{2}$-PMMA and $\mathrm{MoS}_{2}$-PAA materials and evaluation of the $A_{1 g} / 2 \mathrm{LA}$ intensity ratios under $633 \mathrm{~nm}$ excitation. The 2LA mode under $633 \mathrm{~nm}$ excitation is known to be sensitive to crystal defects and lattice disorders and is actively investigated as a diagnostic tool for chemical functionalization. A recent systematic investigation of the $\mathrm{A}_{1 \mathrm{~g}} / 2 \mathrm{LA}$ intensity ratios for defect engineering of ce-MoS $\mathrm{My}_{2}$ byiol coordination showed the potentiality to not only verify the functionalization process, but also allow monitoring of the reactions. ${ }^{18}$ Herein, we acquired Raman spectra from a $20 \times$ $20 \mu \mathrm{m}$ area (196 single spot spectra, in raster mode) of ce-MoS and the calculated average value of the $\mathrm{A}_{1 \mathrm{~g}} / 2 \mathrm{LA}$ intensity ratio was found to be 1.0, with a high homogeneity (Fig. S4, ESI $\dagger$ ). The same process was followed for evaluating the $\mathrm{A}_{1 \mathrm{~g}} / 2 \mathrm{LA}$ intensity ratios for $\mathrm{MoS}_{2}$-PMMA and $\mathrm{MoS}_{2}$-PAA, where the registered average values were found to be 1.29 and 1.21, respectively, also with a high uniformity (Fig. 3c and d). Evidently, covalent functionalization is responsible for the altered intensity of the $\mathrm{A}_{1 \mathrm{~g}}$ and 2LA Raman spectral features. Given the weight loss unveiled by TGA ( $9 \%$ for $\mathrm{MoS}_{2}-\mathrm{PMMA}$ and $8 \%$ for $\mathrm{MoS}_{2}-\mathrm{PAA}$ ), it is obvious that the degree of newly formed covalent bonds is low. It was reported that at the low degree of covalent functionalization via coordination of thiols to S-vacant sites of ce- $\mathrm{MoS}_{2}$, the $\mathrm{A}_{1 \mathrm{~g}} / 2 \mathrm{LA}$ intensity ratio tends to decrease, as compared to ce- $\mathrm{MoS}_{2},{ }^{18}$ while the covalent functionalization of ce- $\mathrm{MoS}_{2}$ via $\mathrm{C}-\mathrm{S}$ bond formation tends to increase it. ${ }^{9}$ Thus, based on our experimental data, we conclude that the major mechanism for the covalent grafting of PMMA and PAA on the surface of ce- $\mathrm{MoS}_{2}$ is the radical-mediated one (mechanism 1 in Fig. 1b), furnishing covalent $\mathrm{C}-\mathrm{S}$ bonds between the carbon backbone of the polymer chain and the surface S-atoms of ce-MoS 2 . However, the possibility of a minor mechanism proceeding via coordination of thiol-terminated polymer chains produced via nucleophilic attack of ce- $\mathrm{MoS}_{2}$ to the thiocarbonylthio $\omega$-terminus of the RAFT-derived polymers cannot be excluded (mechanism 2 in Fig. 1b).

Imaging of $\mathrm{MoS}_{2}-\mathrm{PMMA}$ and $\mathrm{MoS}_{2}$-PAA ensembles with the aid of high-resolution transmission electron microscopy (HRTEM) further validated the presence of single- and few-layered nanosheets covered by organic matter (Fig. 3e and f, respectively). Namely the covalently functionalized nanosheets remained suspended without re-aggregation due to the covalent surface coverage by the polymer chains. Additional images of $\mathrm{MoS}_{2}$-PMMA and $\mathrm{MoS}_{2}$-PAA ensembles as well as of ce- $\mathrm{MoS}_{2}$ are shown in the ESI $\dagger$ (Fig. S5).

The described method allowed us to produce hydrophobic $\mathrm{MoS}_{2}$-PMMA and hydrophilic $\mathrm{MoS}_{2}$-PAA ensembles, furnishing stable dispersions in toluene and water, respectively. 

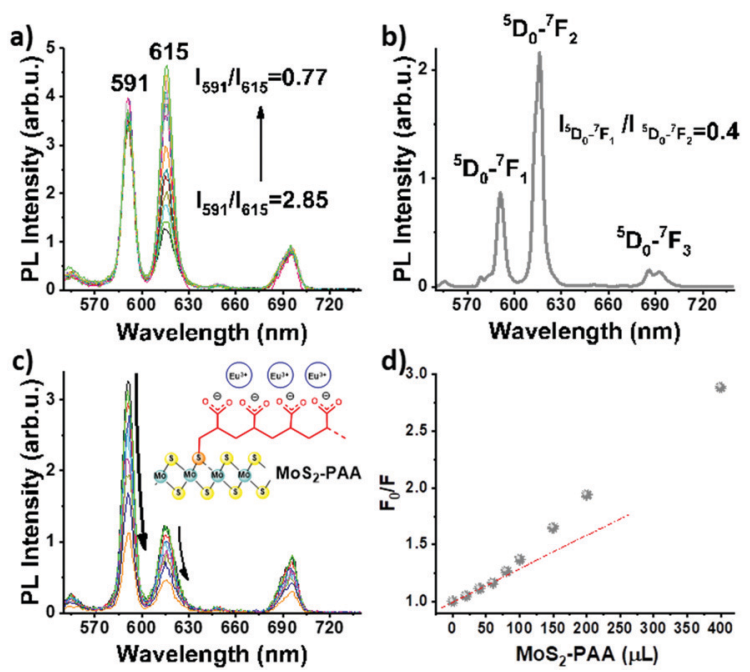

Fig. 4 Fluorescence emission spectra of (a) $\mathrm{Eu}\left(\mathrm{NO}_{3}\right)_{3}$ titrated by aqueous alkaline PAA, (b) Eu(NO$\left.)_{3}\right)_{3}$ in the solid state, and (c) $\mathrm{Eu}\left(\mathrm{NO}_{3}\right)_{3}$ titrated by aqueous alkaline $\mathrm{MoS}_{2}-\mathrm{PAA}$. All spectra were recorded under ambient conditions with excitation at $391 \mathrm{~nm}$. (d) Experimental plot of the change of the $\mathrm{Eu}^{3+}$ fluorescence emission intensity at $591 \mathrm{~nm}$ versus the introduced amount of $\mathrm{MoS}_{2}$-PAA polycarboxylate. The red dashed line highlights the deviation of the experimental data from the linear expression.

The latter enabled the utilization of the polycarboxylic nature of $\mathrm{MoS}_{2}$-PAA nanosheets to evaluate the complexation of watersoluble lanthanide cations. Europium(III) cations were selected as fluorescent probes to monitor the complexation dynamics via fluorescence spectroscopy. A solution of $\mathrm{Eu}\left(\mathrm{NO}_{3}\right)_{3}$ was initially titrated by alkaline PAA (i.e. in the carboxylate form) and the characteristic fluorescence emission peaks of $\mathrm{Eu}^{3+}$ at $591 \mathrm{~nm}\left({ }^{5} \mathrm{D}_{0}{ }^{-} \mathrm{F}_{1}\right.$ transition $)$ and $615 \mathrm{~nm}\left({ }^{5} \mathrm{D}_{0}{ }^{-}{ }^{7} \mathrm{~F}_{2}\right.$ transition $)$ were monitored under $391 \mathrm{~nm}$ excitation. During the addition of the PAA carboxylate, the $615 \mathrm{~nm}$ peak was significantly increased, while the $591 \mathrm{~nm}$ peak was slightly decreased (Fig. 4a). This observation was indicative of the complexation of $\mathrm{Eu}^{3+}$ by the polycarboxylate chains and the formation of an insoluble complex, where the ${ }^{5} \mathrm{D}_{0}{ }^{-}{ }^{7} \mathrm{~F}_{2}$ transition became dominant, similarly to the solid $\mathrm{Eu}\left(\mathrm{NO}_{3}\right)_{3}$ precursor (Fig. 4b). Next, aqueous $\mathrm{Eu}\left(\mathrm{NO}_{3}\right)_{3}$ was titrated by alkaline $\mathrm{MoS}_{2}$-PAA (i.e. in the polycarboxylate form) and the fluorescence emission spectrum of $\mathrm{Eu}^{3+}$ was found to be gradually quenched by the incremental addition of $\mathrm{MoS}_{2}$-PAA (Fig. 4c). To shed more light on the quenching dynamics, we plotted the change of the fluorescence emission intensity at $591 \mathrm{~nm}$ versus the introduced amount of $\mathrm{MoS}_{2}$-PAA polycarboxylate, in analogy to a Stern-Volmer plot. The experimental plot deviates from a linear expression and displays an upward shift, originating from the presence of a static quenching mechanism. ${ }^{19}$ Static quenching is likely to be driven by the strong ground state electrostatic complexation of $\mathrm{Eu}^{3+}$ by $\mathrm{MoS}_{2}$-PAA. This was also corroborated by the change of the $I_{591 \mathrm{~nm}} / I_{615 \mathrm{~nm}}$ intensity ratio, which was found to decrease from 2.85 to $c a .2 .0$ and is also attributed to complexation, and likewise the case of complexation of $\mathrm{Eu}^{3+}$ by the free PAA. Collectively, the covalent $\mathrm{MoS}_{2}-\mathrm{PAA}$ ensembles effectively complexed the lanthanide cations, while the dispersion remained stable in aqueous media.
In summary, we presented a simple one-step synthetic method to obtain well-defined $\mathrm{MoS}_{2}$-polymer nanostructures with tuneable hydrophobicity and hydrophilicity, maintaining the 2D dimensionality and the metallic properties of $\mathrm{MoS}_{2}$. Utilizing the hydrophilic $\mathrm{MoS}_{2}$-PAA ensemble featuring polycarboxylate species, we showed efficient complexation of $\mathrm{Eu}^{3+}$, noting a future use of $\mathrm{MoS}_{2}-\mathrm{PAA} / \mathrm{lanthanide}$ hybrid materials for (opto)electronics. ${ }^{20}$

The TEM study was performed in the Laboratorio de Microscopias Avanzadas (LMA), Universidad de Zaragoza (Spain). R. A. acknowledges support from the Spanish MICINN (PID2019104739GB-100/AEI/10.13039/501100011033), the Government of Aragon (projects DGA E13-20R (FEDER, EU)) and the EU H2020 projects "ESTEEM3" (Grant number 823717) and Graphene Flagship (881603).

\section{Conflicts of interest}

There are no conflicts to declare.

\section{References}

1 A. Stergiou and N. Tagmatarchis, Chem. - Eur. J., 2018, 24, 18246-18257.

2 S. Bertolazzi, M. Gobbi, Y. Zhao, C. Backes and P. Samorì, Chem. Soc. Rev., 2018, 47, 6845-6888.

3 R. Canton-Vitoria, T. Scharl, A. Stergiou, A. Cadranel, R. Arenal, D. M. Guldi and N. Tagmatarchis, Angew. Chem., Int. Ed., 2020, 59, 3976-3981.

4 I. K. Sideri, R. Arenal and N. Tagmatarchis, ACS Mater. Lett., 2020, 2, 832-837.

5 I. K. Sideri, Y. Jang, J. Garces-Garces, A. Sastre-Santos, R. CantonVitoria, R. Kitaura, F. Fernandez-Lazaro, F. D'Souza and N. Tagmatarchis, Angew. Chem., Int. Ed., 2021, 60, 9120-9126.

6 A. Stergiou, C. Stangel, R. Canton-Vitoria, R. Kitaura and N. Tagmatarchis, Nanoscale, 2021, 13, 8948-8957.

7 T. H. Le, Y. Oh, H. Kim and H. Yoon, Chem. - Eur. J., 2020, 26, 6360-6401.

8 D. Voiry, A. Goswami, R. Kappera, C. D. C. C. E. Silva, D. Kaplan, T. Fujita, M. Chen, T. Asefa and M. Chhowalla, Nat. Chem., 2015, 7, 45-49.

9 K. C. Knirsch, N. C. Berner, H. C. Nerl, C. S. Cucinotta, Z. Gholamvand, N. McEvoy, Z. Wang, I. Abramovic, P. Vecera, M. Halik, S. Sanvito, G. S. Duesberg, V. Nicolosi, F. Hauke, A. Hirsch, J. N. Coleman and C. Backes, ACS Nano, 2015, 9, 6018-6030.

10 E. Er, H.-L. Hou, A. Criado, J. Langer, M. Möller, N. Erk, L. M. LizMarzán and M. Prato, Chem. Mater., 2019, 31, 5725-5734.

11 I. Gomez-Munoz, S. Laghouati, R. Torres-Cavanillas, M. MorantGiner, N. V. Vassilyeva, A. Forment-Aliaga and M. GimenezMarques, ACS Appl. Mater. Interfaces, 2021, 13, 36475-36481.

12 H. Cheng, N. Dong, T. Bai, Y. Song, J. Wang, Y. Qin, B. Zhang and Y. Chen, Chem. - Eur. J., 2016, 22, 4500-4507.

13 Z. Souri, M. Adeli and E. Mehdipour, New J. Chem., 2020, 44, 17961-17969.

14 L. Shen, X. Han, J. Qian and D. Hua, RSC Adv., 2017, 7, 10791-10797. 15 G. Moad, E. Rizzardo and S. H. Thang, Polym. Int., 2011, 60, 9-25. 16 H. Willcock and R. K. O'Reilly, Polym. Chem., 2010, 1, 149-157.

17 X. S. Chu, A. Yousaf, D. O. Li, A. A. Tang, A. Debnath, D. Ma, A. A. Green, E. J. G. Santos and Q. H. Wang, Chem. Mater., 2018, 30, 2112-2128.

$18 \mathrm{X}$. Chen, P. Denninger, T. Stimpel-Lindner, E. Spiecker, G. S. Duesberg, C. Backes, K. C. Knirsch and A. Hirsch, Chem. Eur. J., 2020, 26, 6535-6544.

19 D. Genovese, M. Cingolani, E. Rampazzo, L. Prodi and N. Zaccheroni, Chem. Soc. Rev., 2021, 50, 8414-8427.

20 J. C. Bunzli and C. Piguet, Chem. Soc. Rev., 2005, 34, 1048-1077. 